\title{
Green Vehicle Routing Under Customer Demand Uncertainty
}

\author{
Dereje DejeneMengistu, M. Srinivasa Rao, V.V.S.Kesava Rao
}

\begin{abstract}
: customer satisfaction is the main focus area in supply chain management and the distribution of goods plays a vital role in customer satisfaction. cost optimization and in time delivery leads to customer satisfaction. Optimization of Vehicle route plan is the method generally applied to deal it. such plans shall consider the minimization of pollution emissions. This paper proposes a optimization method to handle the vehicle routing problem(vrp). Genetic algorithm and fuzzy clustering algorithm are applied in the method.
\end{abstract}

Keywords: vrp, fuzzy, customer, and emission.

\section{I.INTRODUCTION}

Supply chain management deals with flow of goods from supplier to customer. Supply chain management covers procurement, production, sales and distribution areas. The goods are transported through different transporting modes. Vehicle Routing Problem (VRP), a mode of transport, involves the formulation of a set of routes for a fleet of vehicles, starting and terminating at a depot and serving a set of customers with identified demands. Each customer must be visited by one of these routes and all the customers must be assigned to vehicles such that the restrictions on the capacity of vehicles and the duration of a route are met. Some vehicle routing problems have pre-set time constraints on the periods of the day in which customers should be served. Authors [12] introduced The vehicle routing problem (VRP). VRP has an important practical significance of social benefits and enterprisers benefits. The importance of environmental issues is put into regulation by various governments, which potentially has a tangible impact on supply chain management. Due to this various researches are being done continuously on the interaction between logistics and environmental factors [18] . With the continuous development of commodity economy, it becomes important to process customer orders and deliver them via suitable freight vehicles. customer satisfaction is the main focus area in supply chain management. the distribution of goods plays a vital role in customer satisfaction.

Manuscript received on May 21, 2021.

Revised Manuscript received on September 04, 2021.

Manuscript published on September 30, 2021.

*Correspondence Author

Dereje Dejene Mengistu*, Department of Mechanical Engineering Andhra University College Andhra Pradesh, India. Email:derejed007@gmail.com

M. Srinivasa Rao, DGM Maintnance, Vizag Steel Plant, Visakhapatnam, Andhra Pradesh, India

Prof. V.V.S.Kesava Rao, Department of Mechanical Engineering Andhra University College Andhra Pradesh, India. Email: kesava9999@gmail.com Sciences Publication (BEIESP). This is an open access article under the CC BY-NC-ND license (http://creativecommons.org/licenses/by-nc-nd/4.0/) (c) The Authors. Published by Blue Eyes Intelligence Engineering and

Previous researches on VRP generally take into account the economic factors, such as distance, price, and time windows. And varieties of heuristic algorithm are designed to solve these problems [2]. As per authors [2,3] optimization of the energy consumption and carbon emissions has happened to be interesting topic. Reducing the energy consumption and carbon the objective function emission has great significance on the environment.

With the continuous development of technology and consumer economy, customers are demanding quality in service. If the distributor provides service quality in terms of recognition, delivery terms, packaging etc. customer loyalty towards the enterprise improves. Inappropriate distribution services may lead to loss of customers will and even worse increase the transportation costs of the unit.

To achieve a win-win situation for the vehicle distribution path designing, an appropriate grouping method is proposed to divide the customers into reasonable groups. A feasible route path is designed with in the group for cost optimization. In real life situations the information about parameters, variables may be incomplete and imprecise in nature. The sort of uncertainty is often handled by history of the data based on randomness. but always it may not be true. hence probability cannot be considered. Fuzziness of data is considered in this paper. With the rapid development of on line business and consumerization demand for the goods are ever increasing and speedy and timely delivery has become a requirement to customer satisfaction. This is resulting into lot of exhaust gas emissions and ever increasing pollution levels. grouping of customers may result into lesser distance travel and reduction in pollution levels including reduction in travelling costs. Optimization of the routes of distribution vehicles and reduction of energy consumption and carbon emission in meeting customers demand and satisfaction is the focus of the present paper.

Section 2 discusses literature review. Section 3 provides the explanation of the projected model. Section 4 deals the fuzzy cluster method and GA algorithm to solve the problem. numerical results are mentioned in Section 5. Section 6 deals with future scope of study.

\section{II.LITERATURE REVIEW}

Authors[1] have proposed to solve the optimal distribution route plan of trucks. According to the research of different problems, various types of VRP are formulated and applied mathematical model [4] .Author [5]. applied a tabu search algorithm in vrp problem, To gain optimal results, types of algorithms were designed to solve the problems [6-8] .Authors.[9]applied a large neighborhood search algorithm.

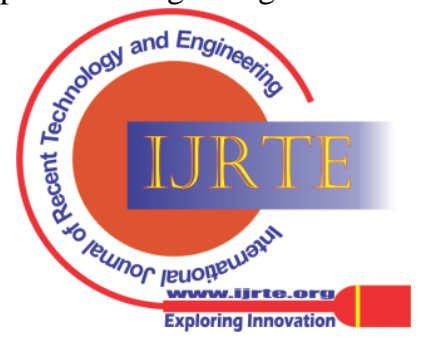




\section{Green Vehicle Routing Under Customer Demand Uncertainty}

Author . [10] proposed a calculation model for vehicle energy consumption, author [11] proposed optimization of the fuel consumption as well as travel time. Author [12] studied the problem of vrp based on time-varying speed. Author [4] proposed the green vehicle routing problem. He [13] established a heterogeneous vehicle routing model by considering the carbon emissions during the vehicle operation. The vehicle routing problem with time windows was proposed by [14] and widely studied by the researchers. Most of the studies focused to solve the vrp problem by Appling techniques such as GA, TS, etc. $[6,15,16]$. Author [17] established a multi objective optimization model for the vehicle routing problem. Authors [2] proposed a time dependent VRP which is considered in the model as a demand attribute.It is proposed in this paper is to group the customers reasonably based on the differences of customer demand.

Then to optimize the costs including vehicle cost, fixed cost, energy consumption, carbon emission, and penalties for deviations in time windows.

\section{MODEL DESCRIPTION}

In this paper, transportation network, is represented by a directed complete graph $\mathrm{M}=(\mathrm{N}, \mathrm{A})$. The vertex set is composed by a depot 0 and a set of customers $\mathrm{N} 0=\mathrm{N} \backslash 0=$ $\{1,2,3, \ldots$.$\} . The number of vehicles is a deterministic$ parameter and represented by $K=\{1,2,3, \ldots$. . $\}$. The capacity of each vehicle is equal to Qe .Each vehicle $\mathrm{K}$ has the earliest departure time ek from the depot after unloaded and the latest arrival time $\mathrm{lk}$ at the depot after finishing the services of customers.

the customer demand $\mathrm{i}$ for goods from the previous order is denoted by $\varphi \mathrm{il}$, and the other demand for different

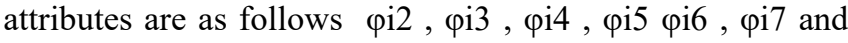
pi8. the 8 attributes into two types, quantitative and qualitative. For the qualitative demand, we use trapezoidal fuzzy number $\left(\alpha \_\mathrm{i} 1^{\wedge}(\sim \mathrm{p}), \alpha \_\mathrm{i} 2^{\wedge}(\sim \mathrm{p}), \alpha \_\mathrm{i} 3^{\wedge}(\sim \mathrm{p}), \alpha \_\mathrm{i} 4^{\wedge}(\sim \mathrm{p}),\right)$ to present five demand levels, in which $\mathrm{i}$ presents the customer number and $\mathrm{p}$ attribute. On the other hand, mathematical method is used to eliminate the dimensional and evaluate the similarity. On this basis, we cluster the customers into different groups, the customers can be clustered into 2 or groups, which can be presented by different shapes, triangle, quadrilateral, and pentagon. And en-route goods delivery paths are designed according to the customers' location and time windows, which decide the delivery sequence. connect each customer with a line and with arrowheads. And in each group, the vehicle should start from the depot (the vehicle remained at the depot) and return to the depot after serving all the in-group customers. Typical figure is depicted showing the details.

Table 1: Evaluation linguistic variables' membership function

\begin{tabular}{|l|l|}
\hline & Triangular fuzzy number \\
\hline Very high & $(0.75,1,1,1)$ \\
\hline high & $(0.5,0.75,0.75,1)$ \\
\hline medium & $(0.25,0.5,0.5,0.75)$ \\
\hline low & $(0.0,0.25,0.25,0.5)$ \\
\hline Very low & $(0.0,0,0.25)$ \\
\hline
\end{tabular}

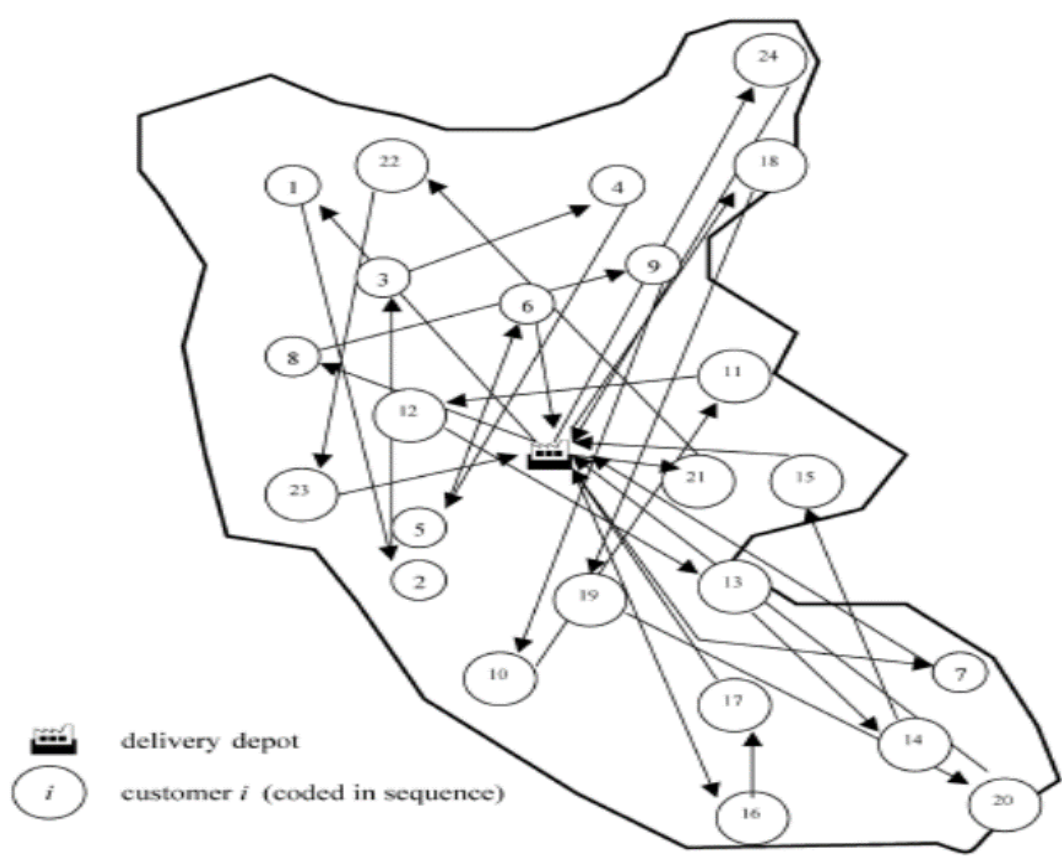

Figure 2: Routing diagram

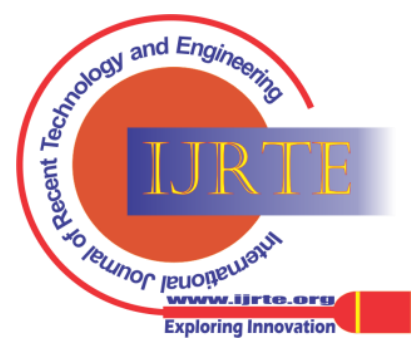


The optimization goal of the problem is to minimize the total cost with vehicle routing optimization.

The optimal objective includes the vehicle fixed costs, time penalty cost, fuel cost, and carbon emissions.

In the next sections, this paper focuses on formulating a two-step model to solve the problem. The first step in model is generated to cluster the customers into groups which ultimately reduces travel distances to determine delivery sequence and the second phase model aims to give out an optimal route en-group which can reduce the energy consumption and emission.

3.1. First step Model. The customers demand attributes consist of several indicators; part of them is quantitative metrics. Others are qualitative metrics. from survey conducted. customer satisfaction mainly depends on the quality of service extended. The eight indicators of decision variables, (1) physical properties, (2) geographical position,
(3) service quality, (4) market value, (5) kind of goods, (6) Good character, and (7) time windows 8) Lifecycle of product. In order to quantify the decision variables, we define the variables in Table 1.

However, the decision variables shall be turned into unit less by standardizing. For modeling convenience, this paper uses the fuzzy clustering method to cluster the customers into appropriate groups, which have the similar demand attributes. Taking into considering the above eight decision variables, the qualitative indicators include $\varphi^{3}, \varphi^{4}, \varphi^{5}$ and $\varphi$ ${ }^{6}$ the other four variables are quantitative indicators.

Based on the language description, we translate the linguistic variables into triangular fuzzy numbers, so that we can calculate the comprehensive similarity between the customers. The linguistic variables represent the correlation between the indicator and customer satisfaction.

Table 1: Definition of customer's demand attributes

\begin{tabular}{|c|c|c|}
\hline Variables & $\begin{array}{l}\text { Variable } \\
\text { name }\end{array}$ & description \\
\hline$\varphi_{i}^{1}$ & $\begin{array}{l}\text { Physical } \\
\text { attributes }\end{array}$ & Number of delivery of goods, weight, volume, etc \\
\hline$\varphi_{i}^{2}$ & $\begin{array}{l}\text { Geographical } \\
\text { attribute }\end{array}$ & Customers' location, place \\
\hline$\varphi_{i}^{3}$ & Service quality & Including response time, service attitude and behavior \\
\hline$\varphi_{i}^{4}$ & Market value & Value of products, generally refers to the market value. \\
\hline$\varphi_{i}^{5}$ & kind of goods & $\begin{array}{l}\text { Types of goods and external similarity with other goods. If the } \\
\text { external similarity is higher, that is easy handle }\end{array}$ \\
\hline$\varphi_{i}^{6}$ & Good character & Fragileness, perishability etc. \\
\hline$\varphi_{\mathrm{i}}^{7}$ & Time window & Present the customer request delivery time limit of time. \\
\hline$\varphi_{i}^{8}$ & Lifecycle of product & similar life cycles tend to be delivered together \\
\hline
\end{tabular}

In this way, each customer's demand indicators can be denoted by fuzzy number which can be denoted as follows:

$\varphi p i=\left[\alpha \_i 1^{\wedge}(\sim p), \alpha \_i 2^{\wedge}(\sim p), \alpha \_i 3^{\wedge}(\sim p), \alpha \_i 4^{\wedge}(\sim p)\right](1)$

We can get the demand attributers from the orders, which will be given by the customers. However, we should transfer the linguistic variables and the quantitative data as standardized data, so that we can use them to calculate the similarity. And the procedure is shown in below.

3.1.1. Processing of Qualitative Decision Variables. In the first step we use the standard deviation transformation to process the qualitative data, which can guarantee the data availability. And for the second step, we introduce the range conversion to standardize the quantitative variables. The constraint (1) can be firstly transformed as follows.

$$
\varphi_{i}^{\sim p}=\left[\alpha_{i 1}^{\sim p}, \alpha_{i 2}^{\sim p}, \alpha_{i 3}^{\sim p}, \alpha_{i 4}^{\sim p}\right], \quad i \in N_{0}
$$

$$
\begin{aligned}
\text { Where } \quad & \varphi_{i}^{\sim p}=\frac{\left|\alpha_{i, t}^{p}, \overline{\alpha_{t, t}^{p}}\right|}{s_{t}^{p}}, \quad i \in N_{0} \\
\overline{\alpha_{l, t}^{p}} & =\frac{\sum_{i=1}^{N} \alpha_{i, t}^{p}}{N}, t=\{1,2,3,4\}, \quad i \in N_{0}
\end{aligned}
$$

However the indicator $\overline{\alpha_{l, t}^{p}}$ is the mean value $S_{t}^{p}$ is the standard deviation, and $|N|$ is the total customer number. The value range of $p=\{3,4,5,6\}$.the standard deviation is calculated as the data is sample.

$$
S_{t}^{p}=\left[\frac{\sum_{i=1}^{N}\left(\alpha_{i, t}^{p},-\overline{\alpha_{l, t}^{p}}\right)^{2}}{|N|-1}\right]^{1 / 2}, t=\{1,2,3,4\},
$$

Then we can calculate the similarity of qualitative decision-making variables with Hemingway's distance method, as follows.

$$
X_{i, j}^{p}=1-\frac{\left|\alpha_{i, t}^{\sim p},-\alpha_{j, t}^{\sim p}\right|}{4}, \quad i \in N_{0}
$$

$X_{i, j}^{p}$ represents the qualitative correlation between the different customers, and the range of the value is during [0, 1]. $X_{i, j}^{p} \quad$ Indicates relationship of attributors $\varphi_{i}^{\sim p}(k)$ and $\varphi_{j}^{\sim p}(k)$.

\subsubsection{Processing of Quantitative Decision Variables.} Quantitative decision variables are determined values. the normalized variables are treated as a kind of special fuzzy number, which can be used to calculate the similarity of the corresponding quantitative decision variables between two customers.

$$
\varphi_{i}^{\sim p}=\frac{\varphi_{i-\min _{1 \leq n \leq|N|}}^{p}\left\{\varphi_{n}^{p}\right\}}{\max _{1 \leq n \leq|N|}\left\{\varphi_{n}^{p}\right\}-\min _{1 \leq n \leq|N|}\left\{\varphi_{n}^{p}\right\}} \text {, for } p
$$

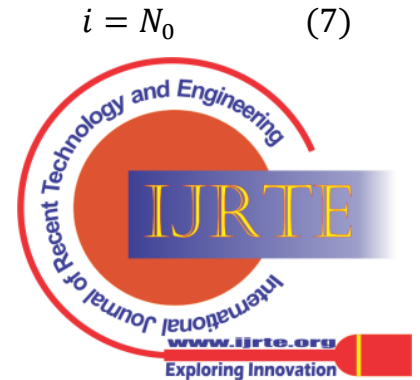




\section{Green Vehicle Routing Under Customer Demand Uncertainty}

$\varphi_{i}^{p}$ and $\varphi_{n}^{p}$ are the actual value of the quantitative decision variables corresponding to customers $\mathrm{i}$ and customers $\mathrm{n}$. is the number of customers. On the basis of the standardization of the quantitative decision, the similarity can be calculated by

$$
X_{i, j}^{p}=1-\frac{\left|\varphi_{i}^{\sim p}-\varphi_{n}^{\sim^{p}}\right|}{\max _{1 \leq n \leq|N|}\left\{\varphi_{n}^{p}\right\}-\min _{1 \leq n \leq|N|}\left\{\varphi_{n}^{p}\right\}} \text {, for } p
$$

Additionally, $\mathrm{p}=1$ represents the weight of the goods; it can determine the number of customer groups. It is used as the termination condition of the customer clustering algorithm in this article. However, the comprehensive similarity for customers is not simply adding upthe similarity for each decision variable. The variables present different influence for the delivery quality. the importance of each decision variable is considered and used a mathematical method to calculate the weight coefficient for each parameter.

3.1.3. Weight Calculation of Decision Variables. After obtaining the order information, the managers (experts) of the distribution center are required to submit evaluation reports about the delivery service index. The experts' evaluation language is generally described as the influence of decision variables on customer satisfaction. the areas of influence are divided into five evaluations, specifically, largest, large, medium, low, and lowest. To calculate the numerical value, the language evaluation is converted to

fuzzy numbers and shown in Table 2.

Based on the importance of the decision variables for customer satisfaction, the weight of the corresponding decision variables can be calculated. For convenience, we denote some key parameters as follows.

And it can be denoted fuzzy number $W u,(u=1,2, \ldots$, $p=1,2, \ldots, m)$ represents the evaluation of decision variables $p$ for decision maker $u$, where $r$ is the total number of decision makers and $m$ is the number of decision variables. And it can be denoted $W_{u, p}\left\{a_{u, p}, b_{u, p}, c_{u, p}, d_{u, p}\right\} . \quad W_{u, p}$ isthe weight value for decision $p$. $Z_{i, p}=\left\{A_{u, p}, B_{u, p}, C_{u, p}, D_{u, p}\right\}(t=$ $1,2, \ldots \ldots,|N|), p=1,2, \ldots, m$ where $|N|$ is the total number of the covering customers. $Z_{i, p}$ represents the comprehensive evaluation index of decision variables $p$ by all decision makers for customer $i$.

$$
\begin{aligned}
& A_{i, p}=\frac{1}{r} \otimes \sum_{u=1}^{r} a_{u, p} \\
& B_{i, p}=\frac{1}{r} \otimes \sum_{u=1}^{r} B_{u, p} \\
& C_{i, p}=\frac{1}{r} \otimes \sum_{u=1}^{r} C_{u, p} \\
& D_{i, p}=\frac{1}{r} \otimes \sum_{u=1}^{r} D_{u, p}
\end{aligned}
$$

Table 2: Membership function of evaluation linguistic variables.

\begin{tabular}{|l|l|l|}
\hline Language terminology & Triangular fuzzy number & Judgment scale \\
\hline largest & $(0.75,1,1,1)$ & 1 \\
\hline large & $(0.5,0.75,0.75,1)$ & 0.75 \\
\hline medium & $(0.25,0.5,0.5,0.75)$ & 0.5 \\
\hline small & $(0.0,0.25,0.25,0.5)$ & 0.25 \\
\hline smallest & $(0.0,0,0.25)$ & 0 \\
\hline
\end{tabular}

The comprehensive evaluation index of decision variables p for customer i can be calculated as
$Z_{i, p}=\frac{1}{r} \otimes \sum_{u=1}^{r} W_{u, p}$

To simplify, we define the comprehensive membership of decision variable $\mathrm{p}$ for customer $\mathrm{i}$ as follows

$Z_{i, p}=\frac{1}{6}\left(A_{i, p}+2 B_{i, p}+2 C_{i, p}+D_{i, p}\right)$

The comprehensive evaluation value of a decision variable $p$ denotes the average value of all customers, such as

$\omega^{\sim p}=\frac{1}{N} \sum_{i=1}^{N} p_{i}$, for $\quad \omega^{p}>0$

Considering that $\omega^{p}$ should satisfy the constraint $\sum_{p=1}^{m} \omega^{p}=1$

$\omega_{p}=\frac{\omega^{\sim p}}{\sum_{p=1}^{m} \omega^{\sim p}}, P=1,2, \ldots, m$

Then, the similarity between customer $i$ and customer $j$ can be denoted as follows:

$$
S_{i, j}=\sum_{p=1}^{m} \omega^{p} X_{i, j}^{p}, i, j \in N_{0}
$$

Finally, we get the comprehensive similarity between customers which can be represented by a similarity matrix just as follows:

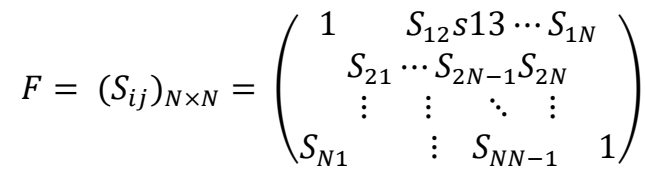

the specific algorithm FCM procedure is summarized as in Algorithm.

To obtain the cluster solutions, we apply the procedure as follows

Step 1: Data initialization, input the fuzzy equivalent matrix $(k)$. Let iterations $\pi=1$.

Step 2: Find out the largest elements in the matrix $(k)$, and chose the customer with the smaller number as the target customer and denote it with $s$.

Step 3: Mark the selected target customer and delete the corresponding row $(k)$. Specifically, once a customer is chosen as the target customer, its column is marked by $(k)$, mark the corresponding elements in row $(k)$ as 0 .

Step 4: Find out the largest element of matrix $(k)$, denoted the similarity value as.

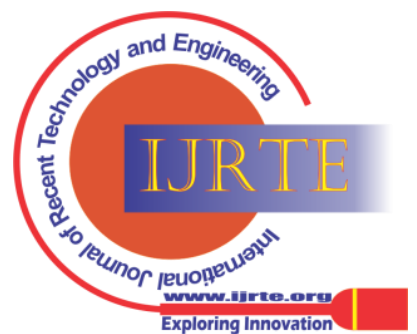


If $\omega_{r s} \leq$ (pre-given threshold value), then go to Step 5 . Otherwise, calculate the total weight of the customers $Q$.

Make judgment, if $Q \geq Q_{l e}$, go to Step 5. Otherwise, put customers $s$ and $r$ in same group, then delete the corresponding row $(k)$ of matrix $(k)$, return to Step 4.

Step 5: Set the customer clustering termination conditions; if the matrix is empty, then clustering process stops; else let $\pi=\pi+1$ and got to Step 2 .

3.2. Second step Model (without speed variation). For the second step in model, should assign the appropriate route for the customer en-groups. In this part we consider the energy conservation and emission reduction and target to reduce the comprehensive costs. At first, regarded the travel speed as fixed variables and establish a conventional model. On this basis, considered the speed as time variation variables and reform the model, which is consistent with the actual situation. To solve the problem, we design a genetic algorithm. To provide a precise statement of this problem, we define the parameters and indices shown in Table 3.For the group route optimization, two decision variables considered as follows:

$X_{i j}^{l^{k}}$

$=\left\{\begin{array}{l}1, \text { if the vehicle } l^{k} \text { travel on the edge }(i, j) \\ 0, \quad \text { otherwise }\end{array}\right.$ $z_{i j}^{l^{k}}$

$=\left\{\begin{array}{l}0, \\ 0,\end{array}\right.$

1, if the vehicle i need to be seviced by the kth vehicle for type $l$ (20)
The model can be formulated as follows for an objective function $U_{R}$ :

$$
\begin{aligned}
\min _{R}=C_{1} \sum_{i \in T_{R}} & \max \left[a_{i}-s_{i}, 0\right]+C_{2} \sum_{i \in T_{R}} \max \left[s_{i}-b_{i}, 0\right] \\
& +\sum_{(i j) \in T_{R}} C_{0} O_{F, i j}\left(Q_{c}^{l}+q_{i j}^{l^{k}}, V_{i j}, d_{i j}\right) \\
& +\sum_{(i j) \in T_{R}} C_{e} \sigma O_{F, i j}\left(Q_{c}^{l}+q_{i j}^{l^{k}}, V_{i j}, d_{i j}\right) \\
& +C_{1}+C_{4} \sum_{i \in T_{R}} \max \left[F r_{i}-F r n_{i}, 0\right] \\
& * q_{i j}^{l^{k}} * \operatorname{pr}_{i}
\end{aligned}
$$

The objective function (20) is to minimize the total routing cost, including fixed cost, energy consumption cost, carbon emission cost, and penalty for time windows

\section{Constraints:}

products which are perishable due to variable factors, such as the metabolism of products, chemical reaction etc. in the process of distribution affect the life. This results in the decrease of customer satisfaction. to make reasonable compensations to customers. The perishability is calculated by adding penalty cost which shall be minimized. It is assumed that customer order required the product life in the distribution center to be $100 \%$. When customer point i was served, the freshness of product Frn $_{\mathrm{i}}$ could be calculated as

\begin{tabular}{|c|c|}
\hline Notations & Detailed Definition \\
\hline$N$ & $\begin{array}{l}\text { Where } N=\{0,1,2, \cdots, n\}, 0 \text { represents the depot, } N 0=N \backslash 0=\{1,2, \cdots, n\} \text { is the set of the customers } \\
\text { which should be delivered. }\end{array}$ \\
\hline A & $A=\{(i, j): i, i \in N\}$ Represents the arcs. \\
\hline $\mathrm{T}_{\mathrm{R}}$ & Set of customers in the same customer group $R$ \\
\hline l & Type of the vehicle. \\
\hline $\mathrm{l}^{\mathrm{k}}$ & $k^{\text {th }}$ vehicle with $l$ type. \\
\hline$Q_{l}^{e}$ & Maximum capacity of each type vehicles \\
\hline$i \in N_{0}$ & Number of customer. \\
\hline$q_{i}$ & Demand of customer'si. \\
\hline $\mathrm{S}_{\mathrm{i}}$ & Time of vehicle arrive at customer $i$. \\
\hline$\left[a_{i}, b_{i}\right]$ & Desired preferred time window for customer i to be serviced. \\
\hline$t_{i}$ & Service time of customer $i$ need. \\
\hline $\mathrm{d}_{\mathrm{ij}}$ & Distance between the customer $i$ and $j$. \\
\hline $\mathrm{V}_{i j}$ & Speed of the vehicle travel on the link $(i, j)$. \\
\hline$q_{i j}^{l^{k}}$ & Load of the vehicle $l k$ travel on the link $(i, j)$. \\
\hline $\mathrm{Q}_{\mathrm{c}}$ & Light weight of the $l$ type vehicle. \\
\hline $\mathrm{C}_{o}$ & Per unit cost of fuel. \\
\hline $\mathrm{Ce}$ & Per unit cost of carbon emissions (carbon tax). \\
\hline$\alpha_{c}$ & Fuel emission factor. \\
\hline$c \mathrm{l}$ & Fixed costs of the vehicle used. \\
\hline$e_{k}$ & Departure time of the vehicle $\mathrm{k}$ from the depot. \\
\hline$l_{\mathrm{k}}$ & Latest arrival time at the depot after services the customers. \\
\hline $\mathrm{C}_{1}$ & Penalty coefficient for earliness arrival. \\
\hline $\mathrm{C}_{2}$ & Penalty coefficient for delay delivery. \\
\hline $\mathrm{C}_{4}$ & Penalty coefficient for freshness decrease \\
\hline$\partial$ & The freshness decreasing coefficient of produce \\
\hline$p r_{i}$ & Price of product to customer i \\
\hline $\mathrm{Fr}_{\mathrm{i}}, \mathrm{Frn}_{\mathrm{i}}$ & Freshness index of expected and new \\
\hline $\mathrm{S}_{\mathrm{i}}$ & Service time of customer $i$. \\
\hline
\end{tabular}
follows:

$$
F r_{i}=\rho^{-\partial\left[\max \left(a_{i}, s_{i}\right)\right]}
$$

Table 3: Sets, indices, and parameters 


\section{Green Vehicle Routing Under Customer Demand Uncertainty}

S.t. $\sum_{l^{k} \in L^{K}} \sum_{j \in T^{R}} X_{0 j}^{l^{k}}=1$

$\sum_{l^{k} \in L^{K}} \sum_{j \in T^{R}} X_{j 0}^{l^{k}}=1$

. Constraint (22) and (23) are guarantee that all service vehicles start from the depot and finally return to the depot for once

$\sum_{l^{k} \in L^{K}} \sum_{j \in T^{R}} X_{i j}^{l^{k}}=1, \forall i \in T_{R}$

Constraint (24) ensures each vehicle travels on the arc $\left(\mathrm{V}_{\mathrm{i}}, \mathrm{V}_{\mathrm{j}}\right)$ no more than once

$$
\sum_{l^{k} \in L^{K}} \sum_{j \in T^{R}} X_{j h}^{l^{k}}-\sum_{\substack{l^{k} \in L^{K} \\ \in T_{R}}} \sum_{j \in T^{R}} X_{h j}^{l^{k}}=0, \quad \forall i
$$

Constraint (25) is the flow balance and shows that the number of the vehicles which arrive at the customer is the same as those which depart

$\sum_{i \in T_{R}} q_{i} \sum_{l^{k} \in L^{K}} \sum_{j \in T^{R}} X_{i j}^{l^{k}} \leq Q_{e}$

Constraint (26) ensures the loading customer demands no more than the vehicle capacity

$a_{i} \sum_{l^{k} \in L^{K}} \sum_{j \in T^{R}} X_{i j}^{l^{k}} \leq S_{i}^{l^{k}} \leq b_{i} \sum_{l^{k} \in L^{K}} \sum_{j \in T^{R}} X_{h j}^{l^{k}}, \quad \forall i \in T_{R}$

Constraint (27) is the time window constraint

$X_{i j}^{l^{k}}\left(S_{i j}^{l^{k}}+t_{i}+\frac{d_{i j}}{v_{i j}}\right) \leq S_{i}^{l^{k}}$

Constraint (28) represents the time limit for vehicle travel. $X_{i j}^{l^{k}} \in\{0,1\}$

Constraint (29) is the binary variable constraint $Y_{i j}^{l^{k}}>0$

Constraint (30) represents the nonnegative of time.

3.3. consideration of speed variations. during different time intervals, the vehicles 'travel speed' is different. The running time is divided into I interval according different extents speed, which can be represented by $\mathrm{T}^{1}, \mathrm{~T}^{2}, \ldots, \mathrm{T}^{\mathrm{I}}$ The mth time interval is indicated as $\left[\mathrm{t}_{\mathrm{m}},+\mathrm{t}_{\mathrm{m}+1}\right]$; in each time interval velocity is constant.

Assume the vehicle derived the customer $\mathrm{i}$ in the $\mathrm{m}$ time interval with the $\operatorname{speed}\left(V_{i j}^{m}\left(l t_{i}\right)\right)$ then the vehicle speed turns $\left(V_{i j}^{m+1}\left(l t_{i}\right)\right)$ in the next time interval, and we denote the vehicle speed as $\left(V_{i j}^{m+p}\left(l t_{i}\right)\right)$ when the vehicle derives the link $(i, j)$. And the distance for the link $(i, j$ ) is denoted by $\left(S_{i j}^{m+1}\left(l t_{i}\right)\right)$. And the total time $\mathrm{tt}_{\mathrm{i}}$ for the vehicle derived across the link $(i, j)$ can be represented by the time function $\mathrm{lt}_{\mathrm{i}} \mathrm{as}$ follows:

$$
t t_{i j}\left(l t_{i}\right)=\frac{d_{j i}^{m}}{V_{i j}^{m}\left(l t_{i}\right)}+\frac{d_{i j}^{m+1}}{V_{i j}^{m+1}\left(l t_{i}\right)}+\cdots \frac{d_{i j}^{m+p}}{V_{i j}^{m+p}\left(l t_{i}\right)}
$$

Considering the variety of the vehicle traveling speed, we can represent the fuel consumption function as follows:

$$
\begin{aligned}
O_{F, i j}\left(Q_{c}^{l}+q_{i j}^{l^{k},}, \overline{V_{l \jmath}}\right. & \left.d_{i j}\right) \\
= & \sum_{q=1}^{I} O_{F, i j}\left(Q_{c}^{l}\right. \\
& \left.+q_{i j}, V_{i j}^{m+q}, d_{i j}^{m+q}\right)
\end{aligned}
$$

If we assumed that the $k$ th vehicle of the $l$ kind begins the task at time, the objective function can be modified as follows with speed variation :

\section{the objective function}

$$
\begin{aligned}
\min _{R}=C_{1} \sum_{i \in T_{R}} & \max \left[a_{i}-s_{i}, 0\right]+C_{2} \sum_{i \in T_{R}} \max \left[s_{i}-b_{i}, 0\right] \\
& +\sum_{(i j) \in T_{R}} C_{0} O_{F, i j}\left(Q_{c}^{l}+q_{i j}^{l^{k}}, V_{i j}, d_{i j}\right) \\
& +\sum_{(i j) \in T_{R}} C_{e} \sigma O_{F, i j}\left(Q_{c}^{l}+q_{i j}^{l^{k}}, V_{i j}, d_{i j}\right) \\
& +C_{1}+C_{4} \sum_{i \in T_{R}} \max \left[F_{i}-F r n_{i}, 0\right] * q_{i j}^{l^{k}} \\
& * \operatorname{pr}_{i}
\end{aligned}
$$

The value of Vij depends on the time interval. The time constraint is represented as follows:

$$
X_{i j}^{l^{k}}\left(S_{i}^{l^{k}}+t_{i}+t t_{i j}\left(S_{i j}^{l^{k}}+t_{i}\right)\right) \leq S_{i}^{l^{k}}
$$

The vehicle service time cannot be earlier than the start time of the distribution center, not later than the time of the delivery center.

$$
t_{1} \leq y_{l^{k}} \leq t_{I}
$$

\section{IV.GENETIC ALGORITHM.}

The above proposed model is a mixed-integer linear programming model. When the PROBLEM size increases, it is difficult to solve the problem with accurate algorithm. However, the GA is appropriate algorithm to solve multivariable complex problem with multiple parameters shows strong robustness. Hence ga is chosen. the key steps for the GA designed as follows.

4.1Encoding. Natural number cod approach is used to characterize chromosomes. Chromosome gene's encoding is based on the results of the customer groups with discontinuous customer numbers. There, 0 expresses the distribution center, and customers are expressed by numbers $1,2,3, \cdots$, N.Different groups are corresponding different chromosomal genes. The beginning and the end of

the genes in the chromosome cod sequence must be " 0 ", on behalf of the vehicle which departs from the distribution center and finally returns to the distribution center after completing the delivery tasks.

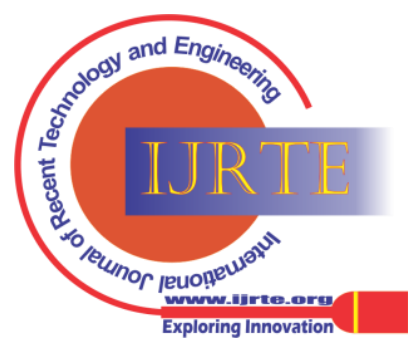


Table 4:Coding of Chromosome

\begin{tabular}{|l|l|l|l|l|l|l|l|l|l|l|}
\hline 0 & 4 & 6 & 5 & 8 & 9 & 3 & 2 & 7 & 1 & 0 \\
\hline
\end{tabular}

4.2. Initial Solution. Construct initial population chromosome cod using $\mathrm{R}$ programming software under Construct initial population chromosome

Fitness Function. In this paper, the objective function value is used to measure the fitness value as follows:

$$
f_{k}=\frac{\left(\mu_{k}-\min \mu\right)}{(\max \mu-\min \mu)}
$$

4.3. Selection Operator. It is individual selection according to tournament selection if the fitness of an individual is denoted by $f k$, then the probability of being selected can be represented by the following:

$$
p_{k}=\frac{f_{k}}{\sum_{k=1}^{k} f_{k}}
$$

4.4. CROSS OVER: the values of the parameters are between the values $0.8 \sim 1$ and the value of the parameter is between the value of $0.2 \sim 0.45$. In this paper, a partial matching crossover strategy is selected, which means that the genes of two crossing points are interchanged by "two points crossing", but the first " 0 " does not participate in the intersection. .pmx cross over is used.

$$
p_{c}=\left\{\begin{array}{r}
p-k \times \frac{f_{\text {max }-f}}{f_{\text {ma }}-\bar{f}}(f \geq \bar{f}) \\
p \quad \text { otherwise }
\end{array}\right.
$$

4.5. Mutation Operator. random exchange of the genes encoding of chromosomes to produce new chromosomes.

4.6. Termination. In this paper, the termination criterions considered as only if the maximum number of iterations is reached, end the algorithm and output the optimal

solution.

The summary of heuristic proposed:

1.Applying fuzzy clustering algorithm for grouping the customers

2.Applying GA for optimization of costs

\section{V.CASE STUDY}

There are 24 customers that should be serviced whose demands can be known in advance, and the customers are supermarkets and the markets in the distribution area. The demand information and location of the customer are shown in Table 6. The data is collected from a local market in Visakhapatnam city. To calculate the cost, we assume that the driving speed of the vehicle is $40 \mathrm{~km} / \mathrm{h}$ for the fixed speed, and the distance between each customer and distribution center is calculated according to their coordinates. The fuel price is $95.55 \mathrm{r} / \mathrm{L}$, and the cost of carbon emission is510 rs/T. The fixed cost of using a vehicle is estimated at Rs 1000 per visit.

\subsection{CUSTOMERS GROUP.}

The customers are clustered into different groups, in which include location, delivery time, physical properties, product value, and service qualities. The corresponding fuzzy evaluation parameters are shown in Table6 and 7. clustering of customers for each vehicle capacity is shown in table 8 and 9.

Table 5: The parameters of the covered vehicles.

\begin{tabular}{|l|l|l|}
\hline parameters & 2T Vehicle & 3T vehicle \\
\hline Gross vehicle weight $w_{z}$ & $2 \mathrm{~T}$ & 2.9 \\
\hline Vehicle emissions $V l$ & 3.2 & 3.6 \\
\hline Type of fuel $f$ & diesel & diesel \\
\hline Load capacity $w_{e}$ & $2 \mathrm{~T}$ & $3 \mathrm{~T}$ \\
\hline The front surface area of a vehicle $S c$ & $2.4 m^{2}$ & $2.65 \mathrm{~m}^{2}$ \\
\hline
\end{tabular}

5.2. Delivery Vehicle route Combining the customer grouping results, we use the designed genetic algorithm to solve the intra group delivery vehicle routing model. Considering that the grouped customers only need one delivery vehicle to complete the delivery service. Set the initial population to 100 , the mutation probabilityis0.09, and the maximum number of iterations was set to 200.Finally, we get the approximate optimal delivery vehicle path plan with time . the energy cost, carbon emission cost, time penalty cost, perishability and fixed cost in the transportation processes objective functions, the distribution vehicle route within the group is optimized. As a heuristic algorithm, the genetic algorithm is generally providing near optimal solution only. Finally, we get the optimal solution shown in Table 10.

5.3. Group Optimization Analysis. The suggestd model takes into account the customer demand and group the customers on the basis of considering the diversity of customer requirements. In order to prove the optimization

effect of the grouping method, this paper solves the case by designing the genetic algorithm without grouping and compares the solution results with the the existing results under the grouping situation table 10 and 11 .

5.4.4. Improve the Quality of Delivery Services. In order to measure the optimization effect of customer grouping on distribution service. the similarity standard deviation of customer demand attribute values within each group evaluates the customer satisfaction and verifies the reasonability of variance does not exhibit.

5.5Analysis of Optimization and Emission Reduction. The energy consumption and carbon emissions are affected by many factors such as road distance ,road condition vehicle load, and vehicle speed.

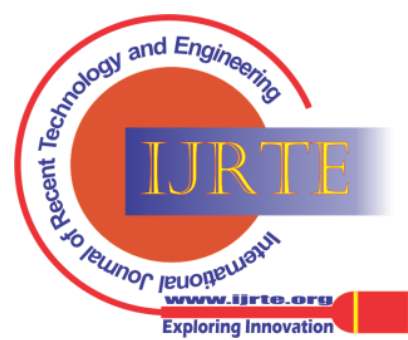




\section{Green Vehicle Routing Under Customer Demand Uncertainty}

The optimal path of each group is solved by the shortest path model and the minimum energy consumption, carbon emission model, perishability minimization respectively. The change of objective function causes the change of fitness function

\section{VI.CONCLUSION AND FURTHER STUDY}

The fastest consumer demand growth requires an efficient distribution planning. Nowadays, special attention is given to routes in which fuel consumption and emissions along with perishability issues can be reduced. The two-step algorithm involving the customer grouping and optimization of cost facilitates to obtain better solution than first in first out customer orders. The improvement is demonstrated in table 13. other meta heuristics can be developed to find out better heuristic.

Table 6: The Demand Information of The Covered Customers.

\begin{tabular}{|c|c|c|c|c|c|c|}
\hline Number & Demand & Distance & $\begin{array}{l}\text { External } \\
\text { similarity }\end{array}$ & $\begin{array}{l}\text { Product } \\
\text { value }\end{array}$ & Time window & $\begin{array}{l}\text { Quality of } \\
\text { Service }\end{array}$ \\
\hline 1 & 0.5 & $(-10.3,9.7)$ & high & medium & {$[8: 00,9.00]$} & highest \\
\hline 2 & 0.2 & $(-4.5,-6.6)$ & low & low & {$[10: 00,12: 15]$} & medium \\
\hline 3 & 0.6 & $(-8.6,6.3)$ & medium & high & {$[12: 00,12: 30]$} & high \\
\hline 4 & 0.4 & $(2.7,9.3)$ & low & medium & {$[10: 00,11: 00]$} & medium \\
\hline 5 & 0.3 & $(-5.3,-4.5)$ & lowest & high & {$[14: 00,14: 30]$} & low \\
\hline 6 & 0.1 & $(-0.6,5.2)$ & low & low & {$[8: 00,9: 30]$} & high \\
\hline 7 & 0.5 & $(18.6,-13.2)$ & high & highest & {$[11: 00,13: 00]$} & high \\
\hline 8 & 0.3 & $(-11.9,3.7)$ & highest & medium & {$[15: 00,16: 30]$} & lowest \\
\hline 9 & 0.1 & $(3.3,7.8)$ & medium & low & {$[14: 00,15: 30]$} & high \\
\hline 10 & 0.1 & $(-1.8,-11.1)$ & medium & medium & {$[13: 00,15: 00]$} & medium \\
\hline 11 & 0.2 & $(8.6,2.5)$ & lowest & lowest & {$[14: 00,14: 30]$} & low \\
\hline 12 & 0.32 & $(-6.9,1.6)$ & medium & high & {$[14: 00,15: 00]$} & medium \\
\hline 13 & 0.39 & $(7.8,-7)$ & high & highest & {$[9: 00,11: 00]$} & medium \\
\hline 14 & 0.2 & $(16.8,-18.4)$ & medium & high & {$[15: 00,16: 00]$} & high \\
\hline 15 & 0.1 & $(12.7,-1.8)$ & low & medium & {$[11: 00,11.30]$} & highest \\
\hline 16 & 0.6 & $(14.3,-21.3)$ & highest & medium & {$[8: 00,11: 00]$} & medium \\
\hline 17 & 0.2 & $(8.2,-15.8)$ & medium & lowest & {$[14: 00,17: 00]$} & lowest \\
\hline 18 & 0.3 & $(11.5,10.4)$ & high & medium & {$[13: 00,14: 30]$} & high \\
\hline 19 & 0.1 & $(1.1,-8.5)$ & medium & highest & {$[10: 00,15: 00]$} & high \\
\hline 20 & 0.4 & $(20.7,-22.7)$ & low & low & {$[11: 00,12.30]$} & Very high \\
\hline 21 & 0.29 & $(6.4,-0.9)$ & high & medium & {$[8: 00,9: 30]$} & low \\
\hline 22 & 0.19 & $(-7.3,11.6)$ & medium & high & {$[13: 00,14: 30]$} & medium \\
\hline 23 & 0.4 & $(-12.2,-2.4)$ & low & low & {$[10: 00,12: 00]$} & medium \\
\hline 24 & 0.5 & $(10.4,21.7)$ & high & high & {$[12: 00,13: 00]$} & very high \\
\hline
\end{tabular}

Table 7: The Qualitative Data of Triangle Fuzzy Evaluation.

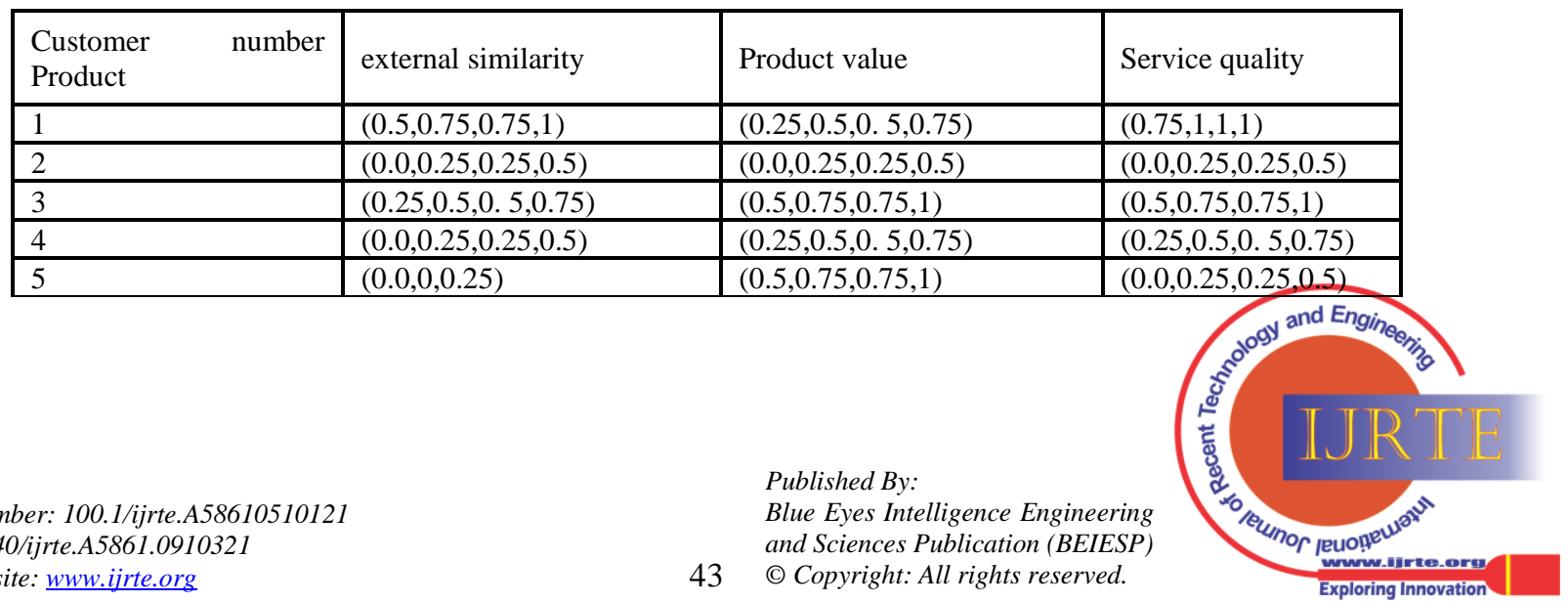




\begin{tabular}{|l|l|l|l|}
\hline 6 & $(0.0,0.25,0.25,0.5)$ & $(0.0,0.25,0.25,0.5)$ & $(0.5,0.75,0.75,1)$ \\
\hline 7 & $(0.5,0.75,0.75,1)$ & $(0.75,1,1,1)$ & $(0.5,0.75,0.75,1)$ \\
\hline 8 & $(0.75,1,1,1)$ & $(0.25,0.5,0.5,0.75)$ & $(0.0,0,0.25)$ \\
\hline 9 & $(0.25,0.5,0.5,0.75)$ & $(0.0,0.25,0.25,0.5)$ & $(0.5,0.75,0.75,1)$ \\
\hline 10 & $(0.25,0.5,0.5,0.75)$ & $(0.25,0.5,0.5,0.75)$ & $(0.25,0.5,0.5,0.75)$ \\
\hline 11 & $(0.0,0,0.25)$ & $(0.0,0,0.25)$ & $(0.0,0.25,0.25,0.5)$ \\
\hline 12 & $(0.25,0.5,0.5,0.75)$ & $(0.5,0.75,0.75,1)$ & $(0.25,0.5,0.5,0.75)$ \\
\hline 13 & $(0.5,0.75,0.75,1)$ & $(0.75,1,1,1)$ & $(0.25,0.5,0.5,0.75)$ \\
\hline 14 & $(0.25,0.5,0.5,0.75)$ & $(0.5,0.75,0.75,1)$ & $(0.5,0.75,0.75,1)$ \\
\hline 15 & $(0.0,0.25,0.25,0.5)$ & $(0.25,0.5,0.5,0.75)$ & $(0.75,1,1,1)$ \\
\hline 16 & $(0.75,1,1,1)$ & $(0.25,0.5,0.5,0.75)$ & $(0.25,0.5,0.5,0.75)$ \\
\hline 17 & $(0.25,0.5,0.5,0.75)$ & $(0.0,0,0.25)$ & $(0.0,0,0.25)$ \\
\hline 18 & $(0.5,0.75,0.75,1)$ & $(0.25,0.5,0.5,0.75)$ & $(0.5,0.75,0.75,1)$ \\
\hline 19 & $(0.25,0.5,0.5,0.75)$ & $(0.75,1,1,1)$ & $(0.5,0.75,0.75,1)$ \\
\hline 20 & $(0.5,0.75,0.75,1)$ & $(0.0,0.25,0.25,0.5)$ & $(0.75,1,1,1)$ \\
\hline 21 & $(0.5,0.75,0.75,1)$ & $(0.25,0.5,0.5,0.75)$ & $(0.0,0.25,0.25,0.5)$ \\
\hline 22 & $(0.25,0.5,0.5,0.75)$ & $(0.5,0.75,0.75,1)$ & $(0.25,0.5,0.5,0.75)$ \\
\hline 23 & $(0.0,0.25,0.25,0.5)$ & $(0.0,0.25,0.25,0.5)$ & $(0.25,0.5,0.5,0.75)$ \\
\hline 24 & $(0.5,0.75,0.75,1)$ & $(0.5,0.75,0.75,1)$ & $(0.75,1,1,1)$ \\
\hline
\end{tabular}

Table 8: The Clustering Results Of Vehicle Restrictions For 2 Tons.

\begin{tabular}{|l|l|l|l|}
\hline Cluster condition & Group No. & Customer No. & volume (tons) \\
\hline \multirow{3}{*}{\begin{tabular}{l}
$Q_{e} \lambda=2 \mathrm{t}$ \\
\cline { 2 - 4 }
\end{tabular}} & Group 1 & $6,21,2,4,17,14,8,22$ & 1.8 \\
\cline { 2 - 4 } & Group 2 & $7,24,15 ;, 1,19,5$ & 2.0 \\
\cline { 2 - 4 } & Group 3 & $9,12,10,3,13,23$ & 1.9 \\
\cline { 2 - 4 } & Group 4 & $11,18,16,20$ & 1.5 \\
\hline
\end{tabular}

Table 9: The clustering results of vehicle restrictions for $\mathbf{3}$ tons.

\begin{tabular}{|l|l|l|l|}
\hline Cluster condition & Group No. & Customer No. & o volume (tons) \\
\hline \multirow{3}{*}{$\begin{array}{l}Q_{e} \\
\lambda=3 \mathrm{t}\end{array}$} & Group 1 & $6,21,2,4,17,14,8,22,5,11$ & 2.5 \\
\cline { 2 - 4 } & Group 2 & $7,24,15,1,19,18$ & 2.0 \\
\cline { 2 - 4 } & Group 3 & $9,12,10,3,13,23,16,20$ & 1.9 \\
\hline
\end{tabular}

Table 10: The Optimization Paths Results With Customer Grouping

\begin{tabular}{|l|l|l|c|}
\hline Group No. & The optimal path en-group & Distance (Km) & Cost \\
\hline Group 1 & $\begin{array}{c}0 \rightarrow 21 \rightarrow 6 \rightarrow 4 \rightarrow 22 \rightarrow 8 \rightarrow 5 \rightarrow 2 \rightarrow 17 \rightarrow 14 \\
\text { (11 } \rightarrow 0\end{array}$ & 109.22 & 4030. \\
\hline Group 2 & $0 \rightarrow 1 \rightarrow 19 \rightarrow 7 \rightarrow 15 \rightarrow 24 \rightarrow 18 \rightarrow 0$ & 104.56 & 3299 \\
\hline Group 3 & $0 \rightarrow 13 \rightarrow 16 \rightarrow 20 \rightarrow 10 \rightarrow 23 \rightarrow 3 \rightarrow 12 \rightarrow 9 \rightarrow 0$ & 110.76 & 3600 \\
\hline
\end{tabular}

Table 11: The Optimization Paths Results With No Grouping

\begin{tabular}{|l|l|c|}
\hline Group No. & The optimal path & $\begin{array}{c}\text { Distribution } \\
\text { quality(tons) }\end{array}$ \\
\hline Path 1 & $0 \rightarrow 3 \rightarrow 1 \rightarrow 22 \rightarrow 6 \rightarrow 9 \rightarrow 4 \rightarrow 18 \rightarrow 10 \rightarrow 21 \rightarrow 0$ & 2.8 \\
\hline Path 2 & $0 \rightarrow 13 \rightarrow 19 \rightarrow 16 \rightarrow 14 \rightarrow 7 \rightarrow 17 \rightarrow 11 \rightarrow 2 \rightarrow 5 \rightarrow 20 \rightarrow 0$ & 3.1 \\
\hline Path 3 & $0 \rightarrow 12 \rightarrow 8 \rightarrow 23 \rightarrow 24 \rightarrow 15 \rightarrow 0$ & 1.7 \\
\hline
\end{tabular}

Table 12: The Data Results Of The Model

\begin{tabular}{|c|c|c|c|}
\hline Group No. & $\begin{array}{l}\text { The optimal path } \\
\text { en-group }\end{array}$ & $\begin{array}{l}\text { Distance } \\
\text { (Km) }\end{array}$ & $\begin{array}{l}\text { Energy } \\
\text { consumption } \\
(\mathrm{L})\end{array}$ \\
\hline Group 1 & $\begin{array}{l}0 \rightarrow 21 \rightarrow 11 \rightarrow 4 \rightarrow 6 \rightarrow 23 \rightarrow 8 \rightarrow 5 \rightarrow 2 \rightarrow 17 \rightarrow \\
14 \rightarrow 0\end{array}$ & 112 & $\begin{array}{l}\text { Carbon } \\
\text { emission } \\
(\mathrm{Kg})\end{array}$ \\
\hline Group 2 & $0 \rightarrow 1 \rightarrow 24 \rightarrow 18 \rightarrow 13 \rightarrow 7 \rightarrow 19 \rightarrow 0$ & 105.2 & 12.35 \\
\hline Group 3 & $0 \rightarrow 13 \rightarrow 20 \rightarrow 16 \rightarrow 10 \rightarrow 22 \rightarrow 3 \rightarrow 12 \rightarrow 9 \rightarrow 0$ & 110.76 & 13.47 \\
\hline
\end{tabular}




\section{Green Vehicle Routing Under Customer Demand Uncertainty}

\section{Table 13: Comparasition of current practice and proposed model}

\begin{tabular}{|l|l|}
\hline criteria & Total cost (Rs) \\
\hline original & 14200 \\
\hline proposed & 10310 \\
\hline saving & 27.4 percent \\
\hline
\end{tabular}

\section{REFERENCES}

1. G. B. Dantzig and J. H. Ramser, "The truck dispatching problem," Management Science, vol. 6, no. 1, pp. 80-91, 1959.

2. C. Cetinkaya, I. Karaoglan, and H. Gokcen, "Two-stage vehicle routing problem with arc time windows: a mixed integer programming formulation and a heuristic approach," European Journal of Operational Research, vol. 230, no. 3, pp. 539-550, 2013.

3. Y. Huang, L. Zhao, T. Van Woensel, and J.-P. Gross, "Time dependent vehicle routing problem with path flexibility," Transportation Research Part B: Methodological, vol. 95, pp. 169195, 2017.

4. B. Golden, A. Assad, L. Levy, and F. Gheysens, "The fleet size and mix vehicle routing problem," Computers \& Operations Research, vol. 11, no. 1, pp. 49-66, 1984.

5. J. Brando "A tabu search algorithm for the heterogeneous fixed fleet vehicle routing problem," Computers \& Operations Research, vol. 38, no. 1, pp. 140-151, 2011.

6. B. Ornbuki, M. Nakamura, and M. Osamu, "A hybrid search based on genetic algorithm and tabu search for vehicle routing," in Proceedings of the _th International Conference on Artificial Intelligence and So_Computing, vol. 7, pp. 176-181, Banff, Canada, 2002.

7. J. Cordeau,M.Gendreau, and G. Laporte, "A tabu search heuristic for periodic and multi-depot vehicle routing problems," Networks, vol. 30, no. 2, pp. 105-119, 1997.

8. G. Y. Tutuncu, "An interactive GRAMPS algorithm for the heterogeneous fixed fleet vehicle routing problem with and without backhauls," European Journal of Operational Research, vol. 201, no. 2, pp. 593-600, 2010.

9. E. Demir, T. Bektas, and G. Laporte, "An adaptive large neighborhood search heuristic for the pollution-routing problem," European Journal of Operational Research, vol. 223, no. 2, pp. 346359, 2012.

10. T. Bektas, and G. Laporte, "The pollution-routing problem," Transportation Research Part B: Methodological, vol. 45, no. 8, pp. 1232-1250, 2011

11. E.Demir,T.Bektas, andG.Laporte,"Thebi-Objective pollution routing problem, "European Journal of Operational Research, vol. 232, no. 3, pp. 464-478,2014.

12. Y.J. Kwon, Y.J.Choi, and D.-H. Lee, "Heterogeneous fixed fleet vehicle routing considering carbon emission," Transportation Research Part D: Transport and Environment, vol. 23, no. 8, pp. 8189, 2013.

13. J. Qian, Fuel emission optimization in vehicle routing problems with time-varying speeds, Lancaster University Management School Department of Management Science,2011.

14. W.P.Savelsbergh,"Local search in routing problems with time windows, "Annals of Operations Research, vol. 4, no. 1-4,pp. 285305,1985 .

15. O.Braysy and M.Gendreau,"Vehicle routing problem with time windows, par tI :route construction and local search algorithms," Transportation Science, vol. 39, no. 1, pp. 104-118,2005.

16. O. B. Gendreau, "Vehicle routing problem with time windows, Part II: meta heuristics," Transportation Science, vol. 39, no. 1, pp. 119$139,2005$.

17. H. I. Calvete, C. Gale, M.J. Oliveros, and B. SanchezValverde, "A goal programming approach to vehicle routing problems with soft time windows," European Journal of Operational Research, vol. 177, no. 3, pp. 1720-1733, 2007.

18. O.Jabali, , T.VanWoensel and A.G Kok,. Analysis of travel times and $\mathrm{CO} 2$ emissions in time-dependent vehicle routing. Production and Operations Management, 21(6), 1060-1074,2012

\section{AUTHOR'S PROFILE}

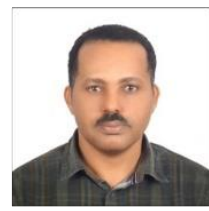

Dereje Dejene Mengistu, received his B.Scdegree in mechanical engineering in 2011 and M.Scdegree in Industrial Engineering from Addis Ababa University, Institute of Technology, Ethiopia in 2014. He is currently a Ph.D. candidate in Andhra University, Visakhapatnam, India. His current area of research interest is optimization of green vehicle routingproblem. He also interests in the development and implementation design, planning and execution methodologies leading to improved supply chain agility.

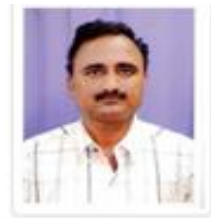

V.V.S. Kesava Rao, received the B.E degree in Mechanical Engineering from Osmania University, India in 1984, M.E, and Ph.D degree from AndhraUniversity, Visakhapatnam, India in 1994, and 2001. He is currently working as a Professor in the department of Mechanical engineering, AU College ofengineering, Visakhapatnam, A.P, India. His research interest is in the filed of industrial Engineering

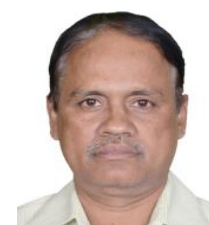

Sri.M.Srinivasa Rao, has obtained M.E (mechanical engg ) and $\mathrm{PhD}$ degree fromAndhra university in the year 2002and2012 respectively. presently working as gm( maintenance ) in Visakhapatnam steel plant and associated with various maintenance activities of rolling mill. The key interesting fields are scheduling , Reliability and optimization of resources

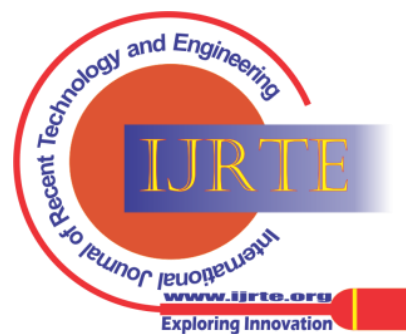

